\title{
Risk of Stroke in Patients With Patent Foramen Ovale Who Had Pulmonary Embolism
}

\author{
Satyajeet Roy, e, Han Le ${ }^{\mathrm{b}}$, Ayobamidele Balogun ${ }^{\mathrm{b}}$, Elizabeth Caskey ${ }^{\mathrm{b}}$, Thomas Tessitore ${ }^{\mathrm{c}}$, \\ Rasagnya Kota ${ }^{c}$, Janice Hejirika ${ }^{c}$, Siyuan $\mathrm{Yu}^{\mathrm{c}}$, Long Nguyen ${ }^{\mathrm{c}}$, Andrew Louis Lazo ${ }^{\mathrm{c}}$, \\ Christopher Yard ${ }^{\mathrm{c}}$, Michael Monaghan ${ }^{\mathrm{c}}$, Menaka Dhingrac \\ Sneha Modic ${ }^{\mathrm{c}}$ Krystal Hunter ${ }^{\mathrm{d}}$
}

\begin{abstract}
Background: The presence of patent foramen ovale (PFO) alone does not increase the risk of ischemic stroke. Several prospective studies with a small number of patients have shown that the risk of ischemic stroke is higher in patients with PFO and pulmonary embolism (PE). We studied the association of ischemic stroke in the patients who had PFO with PE (PFOwiPE) and compared with the patients who had PFO without PE (PFOwoPE).
\end{abstract}

Methods: Electronic medical records of 154 adult patients in our internal medicine office were reviewed. Thirty-four patients had PFOw$\mathrm{iPE}$ and 120 had PFOwoPE. Independent $t$-test and Mann-Whitney U-test were used to compare the continuous variables between the two groups, while Chi-square tests were applied to compare the categorical variables between the two groups. Logistic regression was used for multivariate analysis. The dependent variable was stroke and the independent variable of interest was PFO with, or without PE.

Results: Mean age of patients with PFOwiPE was 54.8 years and patients with $\mathrm{PFOwoPE}$ was 57.8 years $(\mathrm{P}=0.331)$. Mean body mass index (BMI) of the patients with PFOwiPE was significantly greater than the patients with PFOwoPE $\left(32.5 \pm 8.84 \mathrm{~kg} / \mathrm{m}^{2}\right.$ vs. $28.4 \pm 6.99$ $\mathrm{kg} / \mathrm{m}^{2} ; \mathrm{P}<0.05$ ). Mean left ventricular ejection fraction (LVEF) and red blood cell (RBC) count of patients with PFOwiPE was significantly lower than patients with PFOwoPE (LVEF $54.9 \pm 13.01 \%$ vs. $59.6 \pm 6.85 \%, \mathrm{P}<0.05 ; \mathrm{RBC} 4.1 \pm 1.203 \times 10^{6} / \mu \mathrm{L}$ vs. $4.5 \pm 0.596$ $\left.\times 10^{6} / \mu \mathrm{L}, \mathrm{P}<0.05\right)$. There was significantly higher association of congestive heart failure (CHF) in patients with PFOwiPE compared

Manuscript submitted January 17, 2020, accepted January 27, 2020

aDepartment of Medicine, Cooper Medical School of Rowan University, Cooper University Health Care, Camden, NJ, USA

${ }^{b}$ Department of Medicine, Cooper University Health Care, Camden, NJ, USA ${ }^{\mathrm{c} C}$ Cooper Medical School of Rowan University, Camden, NJ, USA

${ }^{\mathrm{d} C o o p e r ~ R e s e a r c h ~ I n s t i t u t e, ~ C o o p e r ~ M e d i c a l ~ S c h o o l ~ o f ~ R o w a n ~ U n i v e r s i t y, ~}$ Camden, NJ, USA

${ }^{\mathrm{e}}$ Corresponding Author: Satyajeet Roy, Department of Medicine, Cooper Medical School of Rowan University, Cooper University Health Care, 1103 North Kings Highway, Suite 203, Cherry Hill, NJ 08034, USA.

Email: roy-satyajeet@cooperhealth.edu

doi: https://doi.org/10.14740/jocmr4094 to patients with PFOwoPE (20.6\% vs. $7.5 \%$; $\mathrm{P}<0.05)$. Association of ischemic stroke was $35.3 \%$ in patients with PFOwiPE and $39.2 \%$ in patients with PFOwoPE. The difference was not statistically significant $(\mathrm{P}=0.682)$.

Conclusions: Association of ischemic stroke was similar in patients with PFOwiPE and patients with PFOwoPE. Association of significantly higher BMI, lower LVEF, lower RBC count, and higher frequency of CHF were associated with patients with PFOwiPE compared to the patients with PFOwoPE.

Keywords: Patent foramen ovale; Pulmonary embolism; Stroke; Cerebrovascular accident

\section{Introduction}

Patent foramen ovale (PFO) is a congenital hole between the right and the left atria. In $15-30 \%$ of the population PFOs tend to persist through adulthood [1-3]. In the majority of the population PFOs remain asymptomatic, but clinical manifestations are believed to be associated with PFOs, such as cryptogenic stroke, especially in patients with atrial fibrillation [4]. This is supported by the fact that the prevalence of PFOs increases to $40-50 \%$ in patients who have cryptogenic stroke, especially before the age of 55 year [4]. The increased risk of ischemic stroke has been reported in several studies in patients with PFOs and acute pulmonary embolism (PE) [5-9]. Although the presence of PFO alone does not increase the risk of stroke [10], nevertheless the risk of recurrent stroke increases with PFOs in patients who had prior cryptogenic stroke [11]. Similarly, the risk of ischemic stroke is shown to be higher in patients with PE and PFO compared to those without PFO, as reported by several studies with small number of subjects [5-8] and a prospective study with a large number of subjects [12]. It is hypothesized that the increase in the right atrial pressure after $\mathrm{PE}$ increases the risk of right-to-left shunt across the PFO resulting in paradoxical embolism and ischemic stroke [11]. In patients with cryptogenic stroke and PFO some studies have reported the presence of asymptomatic PE in 10-37\% of subjects $[13,14]$. We aimed to study whether co-presence of PFO and PE is associated with increased risk of stroke. Our objec- 
tive was to compare the differences in the association of stroke between the patients with PFO and PE and the patients with PFO without PE (PFOwoPE). We hypothesized that the copresence of $\mathrm{PFO}$ and $\mathrm{PE}$ is associated with an increased risk of stroke, and that there are differences in the association of stroke between the patients with PFO and PE and the patients with PFOwoPE.

\section{Materials and Methods}

\section{Study selection}

This study was a retrospective electronic medical record review that compared the differences in the association of stroke between the patients who had PFO with PE (PFOwiPE) and the patients who had PFOwoPE. Patients were seen in our healthcare system between January 1, 2008 and December 31, 2018. The inclusion criteria were adult patients of age 18 years or older who had documentation of PFO in the problem list of their electronic medical records. The exclusion criterion was patients under the age of 18 years and patients who had no PFO.

\section{Data collection}

The following data were collected for each patient: age, gender, race (Caucasian, African American, Hispanic or other), social history (tobacco use, alcohol use, and/or recreational drug use), personal history of deep vein thrombosis or venous thromboembolism (VTE), trauma, surgery, comorbid medical conditions, such as PE, hypertension, diabetes mellitus, hyperlipidemia, hypothyroidism, coronary artery disease, cerebral vascular accident or stroke, migraine, carotid artery stenosis, peripheral arterial disease, congestive heart failure (CHF), atrial fibrillation, chronic obstructive pulmonary disease, asthma, chronic kidney disease, nephrotic syndrome liver disease, hypothyroidism, other endocrine disorders, osteoarthritis and other rheumatologic diseases, colorectal disorder, immunocompromised host secondary to human immunodeficiency virus (HIV) infection, immunodeficiency disorder, or immunosuppressant therapy post-transplant or post chemotherapy, and cancer. We also collected the data on blood pressure, heart rate, body mass index (BMI), lipid profile, serum creatinine, echocardiogram, use of medications, such as estrogen containing medications, oral anticoagulant, aspirin, and statin.

\section{Statistical study}

Collected data were entered into a Microsoft Excel (2016, Redmond, Washington, USA) spreadsheet. Statistical analysis was done using SPSS (Statistical Package for the Social Sciences, version 15.01, IBM, Armonk, New York, USA). Being a retrospective study the rationale behind the sample size depended upon the total number of patients who were documented having a diagnosis of PFO in their problem list with, or without PE between January 1, 2008 and December 31, 2018. A review of the existing literature showed that Le Moigne and colleagues found the rate of stroke in patients with $\mathrm{PE}$ and PFO was $21.4 \%$ [12], while Di Tullio and colleagues found that the rate of stroke in patients with PFO was 6.2\% [10]. Based on the results of these studies a total of 154 subjects were planned for the analysis in order to achieve the sample that would provide $80 \%$ power and $5 \%$ alpha error.

The patients were divided into two groups. The first group represented the patients who had PFOwiPE. The second group represented the patients who had PFOwoPE. Independent $t$ test, or Mann Whitney U-test were used to compare the continuous variables between the two groups. The tests were determined by the distribution of the data. Chi-square test was used to compare the categorical variables between the groups. Multivariate analysis was performed by using logistic regression. The dependent variable was cerebrovascular accident (stroke). The independent variable of interest was PFO with or without PE. Other independent variables that were in the model were determined by the univariate analysis. In this study, significance was defined as a $\mathrm{P}<0.05$.

The study was reviewed and approved by the Institutional Review Board of the Cooper Health System, Camden, New Jersey, USA; and this study was fully compliant with the ethical standards set forth by the CUHC Institutional Review Board.

\section{Results}

A total of 154 patient medical records were included. Among them, 34 (22.1\%) patients had PFOwiPE and 120 (77.9\%) patients had PFOwoPE.

The age range of all the patients with PFO in our study was 19 to 95 years. The majority of the patients with PFO were in the 40 to 69 year age range (Fig. 1). The mean age of the patients in the PFOwiPE group was $54.8 \pm 14.5$ years, and in the patients in the PFOwoPE group was $57.8 \pm 16.0$ years. There was no statistically significant difference in the mean ages between the two groups (Table 1). Gender analysis revealed that the frequency of female patients was significantly higher than the male patients in both the groups $(55.9 \%$ vs. $44.1 \%$ in PFOwiPE, and $54.2 \%$ vs. $45.8 \%$ in PFOwoPE, respectively) ( $\mathrm{P}<0.001)$. There were no statistically significant differences in the proportions of males and females between the two groups (Table 1).

The analysis of race showed that the majority of the patients in both the groups were non-Hispanic Caucasians (67.7\% in PFOwiPE group, and 66.7\% in PFOwoPE group), followed by African Americans (26.5\% in PFOwiPE group, and 20\% in PFOwoPE group), Hispanics (2.9\% in PFOwiPE group, and $8.3 \%$ in PFOwoPE group) and other races (2.9\% in PFOwiPE group, and 5\% in PFOwoPE group) (Table 1). Although there were significantly more non-Hispanic Caucasians in both the groups (PFOwiPE 67.7\% vs. PFOwoPE 66.7\%) compared to the other race categories, the differences were not statistically significant between the two groups.

Social factor analysis showed that a significantly lower 


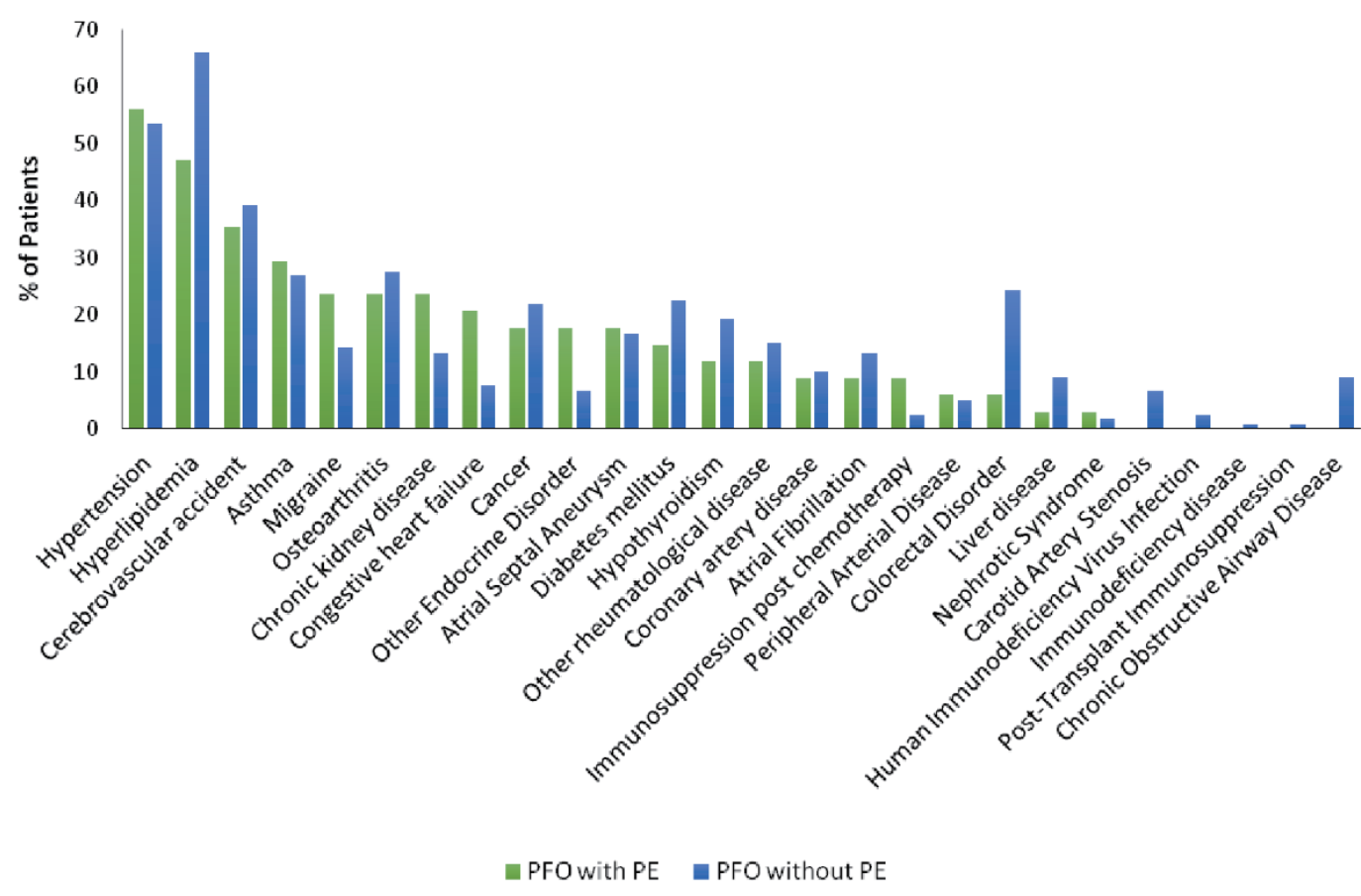

Figure 1. Frequencies of comorbid conditions in patients with patent foramen ovale (PFO) with pulmonary embolism (PE) and in patients with PFO without PE.

percentage of consumption of alcohol in the PFOwiPE group (17.6\%) compared to the PFOwoPE group $(41.7 \%)(\mathrm{P}<0.05)$. Cigarette smoking was associated in about one-third of the patients in both the groups $(29.4 \%$ in PFOwiPE vs. $37.5 \%$ in PFOwoPE), but there was no statistically significant difference between the two groups (Table 1).

There was a significantly higher association of history of VTE in the PFOwiPE group (67.6\%) compared to the PFOwoPE group $(5.8 \%)(\mathrm{P}<0.001)$; but there were no statistically significant differences in the association of history of trauma (20.6\% in PFOwiPE group vs. 15.8\% in PFOwoPE group) and history of surgery $(82.4 \%$ in PFOwiPE group vs. $80.8 \%$ in PFOwoPE group) between the two groups (Table 1).

The mean BMI of the patients with PFOwiPE was significantly greater than the patients with PFOwoPE (32.5 \pm 8.84 $\mathrm{kg} / \mathrm{m}^{2}$ vs. $\left.28.4 \pm 6.99 \mathrm{~kg} / \mathrm{m}^{2} ; \mathrm{P}<0.05\right)$. There was no statically significant difference in the vital signs, such as heart rate, systolic and diastolic blood pressures, between the two groups (Table 2). Similarly, there were no statically significant differences in the lipid parameters and serum creatinine levels between the two groups (Table 2).

We found that the mean red blood cell (RBC) count was significantly lower in the PFOwiPE group $\left(4.1 \pm 1.203 \times 10^{6} /\right.$ $\mu \mathrm{L})$ compared to the PFOwoPE group $\left(4.5 \pm 0.596 \times 10^{6} / \mu \mathrm{L} /\right.$ $\mu \mathrm{L})(\mathrm{P}<0.05)$ (Table 2).

Transthoracic echocardiographic findings showed that the mean left ventricular ejection fraction (LVEF) of the patients with PFOwiPE was significantly lower than the patients with PFOwoPE $(54.9 \pm 13.01 \%$ vs. $59.6 \pm 6.85 \%$; $\mathrm{P}<0.05)$. There was no significant difference in the mean pulmonary arterial pressure between the two groups $(27.3 \pm 10.073 \mathrm{~mm} \mathrm{Hg}$ in PFOwiPE group vs. $27.4 \pm 8.329 \mathrm{~mm} \mathrm{Hg}$ in PFOwoPE group (Table 2). There were only small associations of atrial septal aneurysms and right atrial dysfunction in both the groups $(17.6 \%$ in PFOwiPE group vs. $16.7 \%$ in PFOwoPE group; and $2.9 \%$ in PFOwiPE group vs. $4.2 \%$ in PFOwoPE group, respectively). The differences were not statistically significant (Table 2).

We found that hypertension and hyperlipidemia were the most commonly associated comorbid conditions in the patients in both the groups $(55.9 \%$ in PFOwiPE group vs. $53.3 \%$ in PFOwoPE group; and $47.1 \%$ in PFOwiPE group vs. $65.8 \%$ in PFOwoPE group, respectively). Although the difference for association of hypertension was not statistically significant ( $P$ $=0.792$ ), there was a significantly lesser association of hyperlipidemia in the PFOwiPE group $(\mathrm{P}<0.05)$ (Table 3). Among the patients in the PFOwiPE group, there was a significantly higher association of CHF compared to the patients in the PFOwoPE group (20.6\% vs. 7.5\%; P < 0.05) (Table 3, Fig. 1).

There were no significant differences in the association of other comorbid conditions between the two groups, such as asthma, migraine, osteoarthritis, other rheumatologic diseases, chronic kidney disease, cancer, hypothyroidism, other endocrine disorders, diabetes mellitus, coronary artery disease, atrial fibrillation, immunosuppression post chemotherapy, peripheral arterial disease, colorectal disorders, liver disease, nephrotic syndrome, carotid artery stenosis, HIV infection, immunodeficiency diseases, post-transplant immunosuppression, and chronic obstructive airway disease (Table 3 ).

In our study, the association of ischemic stroke was $35.3 \%$ in the patients with PFOwiPE and 39.2\% in the patients with 
Table 1. Baseline Characteristics

\begin{tabular}{|c|c|c|c|}
\hline Variable & PFO with PE $(n=34)$ & PFO without PE $(n=120)$ & $\mathbf{P}$ \\
\hline Age, mean (SD) & $54.8(14.5)$ & $57.8(16.0)$ & $0.331^{\mathrm{a}}$ \\
\hline \multicolumn{4}{|l|}{ Gender } \\
\hline Female (n, \%) & $19(55.9)$ & $65(54.2)$ & $0.859^{\mathrm{b}}$ \\
\hline \multicolumn{4}{|l|}{ Race } \\
\hline Hispanic (n, \%) & $1(2.9)$ & $10(8.3)$ & $\mathrm{NS}^{\mathrm{b}}$ \\
\hline Other $(n, \%)$ & $1(2.9)$ & $6(5.0)$ & $\mathrm{NS}^{\mathrm{b}}$ \\
\hline \multicolumn{4}{|l|}{ Social factors } \\
\hline Alcohol (n, \%) & $6(17.6)$ & $50(41.7)$ & $<0.05^{\mathrm{b}}$ \\
\hline VTE (n, \%) & $23(67.6)$ & $7(5.8)$ & $<0.001^{\mathrm{b}}$ \\
\hline Trauma (n, \%) & $7(20.6)$ & $19(15.8)$ & $0.514^{\mathrm{b}}$ \\
\hline Surgery (n, \%) & $28(82.4)$ & $97(80.8)$ & $0.841^{\mathrm{b}}$ \\
\hline
\end{tabular}

andependent $t$-test. ${ }^{\text {b}}$ Chi-square and Fisher exact test. PFO: patent foramen ovale; PE: pulmonary embolism; SD: standard deviation; NS: not significant; VTE: venous thromboembolism.

PFOwoPE. We found that the difference was not statistically significant $(\mathrm{P}=0.682)$ (Table 3 , Fig. 1).

Among the medication usage, there were no significant differences in the use of aspirin, statin and estrogen between the two groups. Oral anticoagulant use was significantly higher in the PFOwiPE group compared to the PFOwoPE group
(55.9\% vs. $15.0 \%)(\mathrm{P}<0.001)($ Table 4$)$.

\section{Discussion}

We had several major findings. First, there was no difference in

Table 2. Vitals and Diagnostic Tests

\begin{tabular}{|c|c|c|c|}
\hline Variable & PFO with PE $(n=34)$ & PFO without PE $(n=120)$ & $\mathbf{P}$ \\
\hline Heart rate (beats per minute) (mean, SD) & $77.6(14.732)$ & $74.4(14.036)$ & $0.245^{\mathrm{a}}$ \\
\hline Systolic blood pressure (mm Hg) (mean, SD) & $125.9(17.917)$ & $125.4(17.776)$ & $0.884^{\mathrm{a}}$ \\
\hline Body mass index $\left(\mathrm{kg} / \mathrm{m}^{2}\right)$ (mean, $\left.\mathrm{SD}\right)$ & $32.5(8.848)$ & $28.4(6.994)$ & $0.005^{*}$, a \\
\hline Total cholesterol (mg/dL) (mean, SD) & $163.8(40.739)$ & $163.8(44.291)$ & $1.000^{\mathrm{a}}$ \\
\hline HDL cholesterol (mg/dL) (mean, SD) & $55.2(13.221)$ & $53.3(16.064)$ & $0.519^{\mathrm{a}}$ \\
\hline Triglyceride (mg/dL) (mean, SD) & $103.3(67.503)$ & $108.8(61.660)$ & $0.651^{\mathrm{a}}$ \\
\hline Red blood cells $\left(\times 10^{6} / \mu \mathrm{L}\right)($ mean, SD) & $4.1(1.203)$ & $4.5(0.596)$ & $0.031 *$ a \\
\hline Serum creatinine $(\mathrm{mg} / \mathrm{dL})$ (mean, $\mathrm{SD})$ & $1.2(1.437)$ & $0.9(0.447)$ & $0.272^{\mathrm{a}}$ \\
\hline Left ventricular ejection fraction (\%) (mean, SD) & $54.9(13.005)$ & $59.6(6.848)$ & $0.049 *, a$ \\
\hline
\end{tabular}

${ }^{*} \mathrm{P}<0.05$. alndependent $t$-test. ${ }^{b}$ Chi-square and Fisher exact test. PFO: patent foramen ovale; PE: pulmonary embolism; SD: standard deviation; LDL: low-density lipoprotein; HDL: high-density lipoprotein; PA: pulmonary artery. 
Table 3. Associated Comorbid Conditions

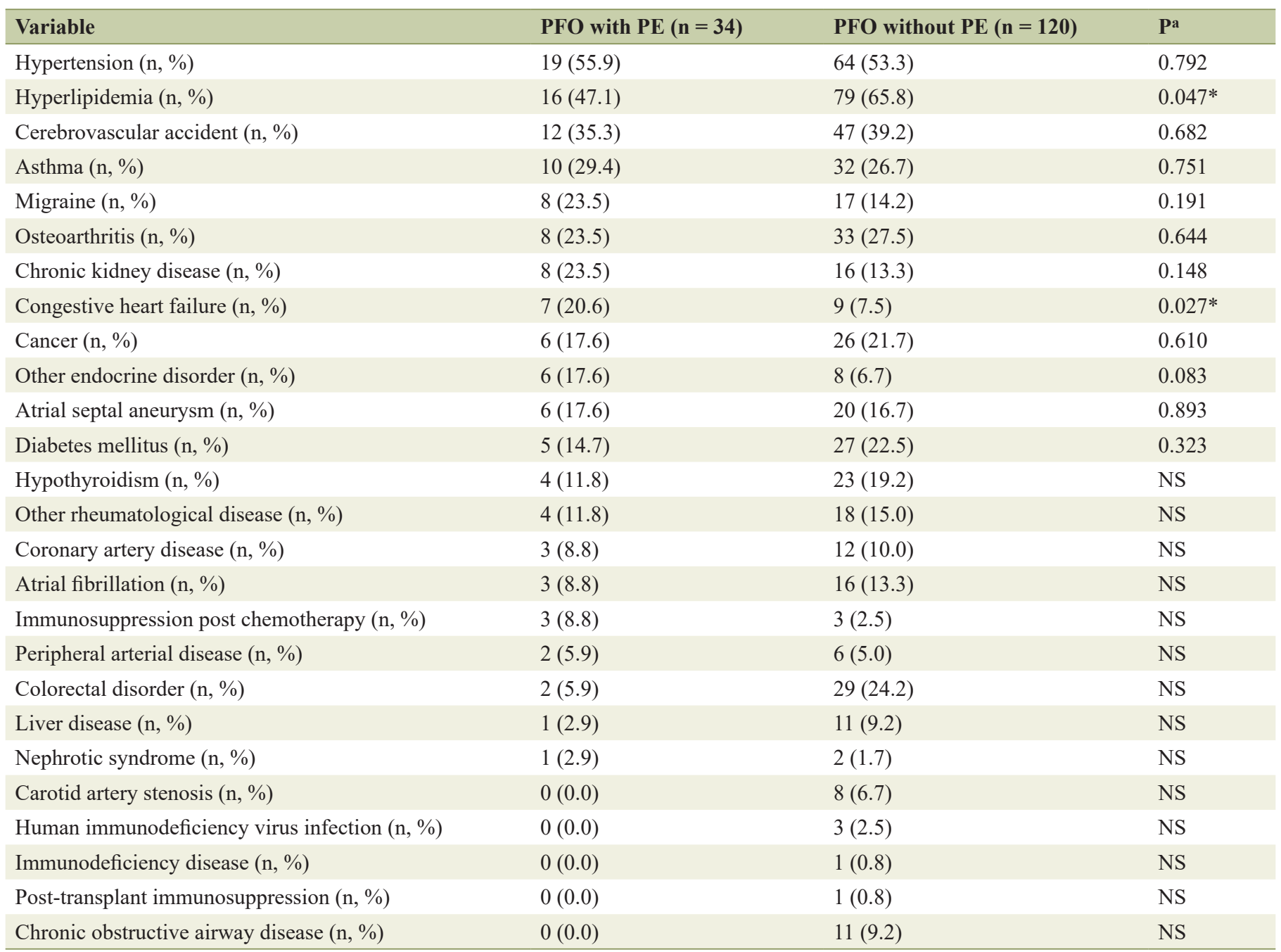

${ }^{*} \mathrm{P}<0.05$. ${ }^{a}$ Chi-square and Fisher exact test. PFO: patent foramen ovale; PE: pulmonary embolism; NS: not significant.

the association of stroke between the patients with PFOwiPE and the patients with PFOwoPE. Many studies have concluded that the patients with PFO are at an increased risk of stroke, as a thrombus that would usually pass into the lungs can transfer between the atria and instead travel to the brain [15-19]. Doyen and colleagues found $17.1 \%$ prevalence of ischemic stroke in their series of 41 patients who had acute intermediate-risk PE and PFO confirmed by contrast transesophageal echocardiography (TEE) [5]. Goliszek and colleagues detected ischemic stroke by magnetic resonance imaging (MRI) in four patients (7.3\%) out of 55 patients who had right ventricular dysfunction (RVD) and intermediate-risk PE, and none in patients with PFO without RVD, suggesting a significantly higher risk of stroke in patients with PFOwiPE compared to the patients with PFOwoPE [6]. Clergeau and colleagues assessed the rate of clinically apparent and silent stroke in patients with $\mathrm{PE}$ with or without PFO. In a series of 60 patients, they found that with PE the frequency of silent strokes in patients with PFO was sig-

Table 4. Medications

\begin{tabular}{llll}
\hline Variable & PFO with PE $(\mathbf{n = 3 4 )}$ & PFO without PE $(\mathbf{n}=\mathbf{1 2 0})$ & Pa \\
\hline Oral anticoagulant (n, \%) & $19(55.9)$ & $18(15.0)$ & 0.001 \\
Statin (n, \%) & $13(38.2)$ & $61(50.8)$ & 0.194 \\
Aspirin (n, \%) & $11(32.4)$ & $61(50.8)$ & 0.057 \\
Estrogen (n, \%) & $1(2.9)$ & $8(6.7)$ & 0.685 \\
\hline
\end{tabular}

${ }^{a}$ Chi-square and Fisher exact test. PFO: patent foramen ovale; PE: pulmonary embolism. 
nificantly higher than that in the patients without PFO (five of $15(33.3 \%)$ vs. one of 45 patients $(2.2 \%), \mathrm{P}=0.003)$ [7]. Similarly, Vindis and colleagues followed 78 patients with acute $\mathrm{PE}$ for a period of 12 months and found that $64.5 \%$ patients with PFO had ischemic brain lesions on baseline MRI compared to $40.4 \%$ in patients without PFO [8]. They found no significant difference at baseline. While at 12-month follow-up MRI, they found that new ischemic brain lesions were significantly higher in the patients with PFO compared to the patients without PFO (33.3\% vs. $5.4 \%, \mathrm{P}=0.008)$ [8]. They concluded that the presence of PFO was associated with new stroke events in patients with PE despite effective anticoagulation therapy [8]. In a prospective cohort of patients with PE, Le Moigne and colleagues reported that the frequency of ischemic stroke was higher in patients with PFO (21.9\%; nine of 42 patients) compared to the patients without PFO $(5.5 \% ; 15$ of 273 patients) [12]. They concluded that in patients with symptomatic PE the frequency of stroke was higher in patients with PFO than that in patients without PFO.

In our study, the association of ischemic stroke was 35.3\% in the patients with PFOwiPE and $39.2 \%$ in the patients with PFOwoPE. Our finding of $35.3 \%$ frequency of stroke in the patients with PFOwiPE surpasses the frequencies observed in the studies as mentioned above, which range from $7.3 \%$ to $33.3 \%$ $[5-8,12]$. On retrospective data review, we did not find a specific association that could attribute for a higher frequency seen in our group of patients.

Many studies have proposed various risk factors that may be associated with increased risk of stroke in patients with $\mathrm{PFO}$ with or without PE, such as paradoxical embolism via right-toleft shunt at rest $[11,20]$, or during Valsalva [21], coexisting atrial septal aneurysm [22], RVD [6], and recurrent paradoxical embolism in presence of PE [16]. On the contrary, other studies did not confirm these findings $[23,24]$. Poulin and colleagues suggest that PFOs should be closed in certain circumstances in order to prevent the right-to-left paradoxical shunting event from occurring [25]. At the same time it is worth considering that not all PFOs are of the same size. Smaller PFOs have less blood flow and therefore a lower chance of a thrombus crossing [26, 27]. Interestingly, we found an even higher frequency of association of stroke in our group of patients with PFOwoPE (39.2\%), but the difference was not statistically significant $(\mathrm{P}=0.682)$.

There are controversial findings regarding the association of PFO with the first stroke and with the recurrent strokes [22, $23,28,29]$. So far the comprehensive analysis of data from multiple case-control studies conducted by Overell and colleagues offers the best evidence regarding a positive association of coexisting PFO/atrial septal aneurysm and stroke (odds ratio (OR): 15.59, 95\% confidence interval (CI), 2.83 - 85.87) [28]. Our data contrast with the previously reported data regarding PFO and PE. We found that the presence or absence of PE alone in patients with PFO does not appear to be an independent risk factor for ischemic stroke. Our findings are supported by smaller studies, such as Sipola and colleagues [30] and Tanislav and colleagues [31]. Both the studies did not report a significant relationship between $\mathrm{PE}, \mathrm{PFO}$, and stroke. The presence of PE within the pulmonary system could result in increased pressures within the pulmonary vasculature lead- ing to increased right-to-left shunting has not been proven despite numerous studies attempting to link PFO, PE and stroke. One explanation for our findings is that patients with PFO may be at higher risk for stroke regardless of PE history [32]. While it is true that one mechanism of stroke in patients with PFO is attributed to paradoxical embolization, it is unlikely for paradoxical embolization to occur without multiple other factors. Some of these factors include a Chiari network and prominent eustachian valve in the right atrium, which are anatomical structures that have been shown to direct blood towards the PFO [32]. Other factors shown to be associated with PFO and stroke include larger PFO size and as polymorphisms in APOC3, a lipoprotein critical in triglyceride metabolism [32]. Yasaka and colleagues found that only $3.2 \%$ of stroke patients with PFO definitely fit the criteria for paradoxical brain embolism [33]. Studies have shown that other causes of stroke, besides paradoxical brain embolism, should be considered in stroke patients with PFO [33]. While the risk of stroke may be increased with PFO, the presence or absence of PE cannot be linked to the increased risk of stroke, as demonstrated by our study. Our study is unique in demonstrating an equivalent frequency of stroke in patients with PFO regardless of the presence of PE.

Second, we found that the BMI was significantly higher in the patients with PFOwiPE compared the patients with PFOwoPE. Not much is known about the role of BMI in patients with $\mathrm{PFO}$ and PE. Obesity is an independent risk factor for PE. Stein and colleagues determined an OR of 2.21 for PE amongst obese patients [34]. A 2008 meta-analysis by Ageno and colleagues, which examined a cohort of over 63,000 patients from 21 case-control and cohort studies also found a VTE risk of 2.33 in obese patients [35]. In subjects with BMI greater than $35 \mathrm{~kg} / \mathrm{m}^{2} \mathrm{Kabrhel}$ and associates showed a six-fold increase in the risk for PE [36]. Their prospective cohort study of 83,766 women demonstrated an increase in unprovoked PE relative risk of $8 \%$ for every point increase in BMI above normal, even in the absence of obesity [36]. However, a 2008 German registry study determined that there was no association between obesity and recurrent VTE [37]. Similarly, Tromso study determined that abdominal obesity rather than BMI alone is a key independent risk factor for VTE [38].

Rosenfeld and colleagues reported significantly greater numbers in patients with PE in the overweight, obese, and morbidly obese categories [39]. In our study, we found similar results. Mean BMI of our patients with PFO and PE was $32.5 \mathrm{~kg} / \mathrm{m}^{2}$ and for patients with PFOwoPE the mean BMI was $28.4 \mathrm{~kg} / \mathrm{m}^{2}$. Obesity is most likely correlated with thrombotic events due to reduced mobility, decreased venous return, and a hypercoagulable state attributed to increased fibrinogen, plasminogen activator inhibitor (PAI-1), factor VII and VIII [40]. PAI-1 is synthesized in adipose tissue, and elevated levels inhibit the removal of fibrin, and can lead to thrombosis. PAI-1 levels have been positively correlated with obesity and elevated BMI. Elevated PAI-1 levels have been discovered in patients with VTE and cerebrovascular accident (CVA) due to increased expression and synthesis in adipose tissue [40]. Therefore, the increased levels of PAI-1 found in obesity may predispose to microvascular and macrovascular, arterial and venous thrombosis [40]. Although there are no previous stud- 
ies that investigated the impact of BMI on patients with $\mathrm{PFO}$ and $\mathrm{PE}$ versus patients with $\mathrm{PFO}$ only, some studies highlight the effect of BMI on patients with hereditary hypercoagulability and PFO-related cryptogenic stroke. Patients with PFrelated cryptogenic strokes and hereditary hypercoagulability tend to have a higher BMI than those with PFO-related cryptogenic stroke and no hereditary hypercoagulable states [41]. Our study concludes that increased BMI remains a significant contributor to $\mathrm{PE}$ in patients who present with a PFO.

Third, we found that the patients with PFO and PE had a significantly higher association of decreased LVEF and CHF in comparison to the patients who had PFOwoPE. Howell and colleagues demonstrated that VTE risk increased dramatically with decreased LVEF [42]. Patients with reduced LVEF are in a hypercoagulable state, which places them at an increased risk of PE [43], likely due to slow, stagnant blood flow in cardiac chambers with irregular and poor contractility predisposing patients to an increased risk of thrombi and emboli formation [44]. Dries and associates demonstrated that ejection fraction is an independent risk factor, similar to atrial fibrillation, for thromboembolic events [44]. Their findings also showed that in women, a $10 \%$ decline in ejection fraction was associated with a $53 \%$ increased risk of thromboembolic events [44]. A logistic regression model in the study conducted by Howell and associates demonstrated that the risk of PE increased in an LVEF-dependent manner, especially in patients with LVEF less than $20 \%$ who had a marked increased risk of developing PE [42]. Similarly, CHF has been previously studied as an independent risk factor for PE, both due to the overlap in risk factors for CHF and VTE, as well as the contribution of CHF to elements of Virchow's triad such as stasis and possible development of a hypercoagulable state [45]. Goliszek and associates found that right sided heart failure as a result of a PE could lead to right-to-left shunting via the PFO, ultimately increasing the risk for ischemic stroke [6]. Several studies have established $\mathrm{CHF}$ as a risk factor for both $\mathrm{PE}$ and stroke, which is consistent with our findings [46, 47]. This is not surprising given that the relative risk of $\mathrm{PE}$ is estimated to be greater than two times higher for patients with CHF compared to those without CHF. Our results correspond with existing data supporting that patients who had PE had an increased association with CHF and with decreased LVEF [48]. Given the numerous pathophysiological links between $\mathrm{CHF}$, reduced LVEF and thromboembolic events, we believe our conclusions are supported by the current data.

Fourth, we found that the patients with PFO and PE had a significantly lower association with hyperlipidemia compared to the patients with PFOwoPE, which is supported by several studies that have determined no link between hyperlipidemia and VTE. While hyperlipidemia and atherosclerosis are wellestablished risk factors for arterial thrombotic events, their link to VTE is not clearly established. Ray and colleagues noted disparate and equivocal data from previous studies regarding the relationship between hyperlipidemia and VTE, in the form that different measures, such as total cholesterol, triglycerides, low-density lipoprotein (LDL), and high-density lipoprotein (HDL) levels did not demonstrate consistent relationships to the incidence of VTE [49]. McColl and colleagues compared 62 women with VTE with 98 healthy age-matched controls, and observed similar findings like in our study [50]. Total serum cholesterol and mean LDL levels were statistically lower among women with VTE compared to controls [50]. Lipids are known to decline during an acute vascular event, such as VTE or myocardial infarction [49]. This can cause a potential negative confounding effect between dyslipidemia and VTE [49]. The MEGA study examined 2,234 patients and determined no relationship between any lipid levels and VTE except that decreasing levels of apolipoproteins B and A1 were associated with increased risk of venous thrombosis [51]. We believe that the lower association of hyperlipidemia in the patients with $\mathrm{PFO}$ and $\mathrm{PE}$ is a unique finding in our study, which requires further studies to establish a firm association.

Fifth, we found that the mean RBC count was significantly lower in the patients with $\mathrm{PFO}$ and $\mathrm{PE}$ relative to the patients with PFOwoPE. Elevated RBC count, such as in polycythemia vera, is associated with thrombosis in approximately $20 \%$ of the patients; while low RBC count seems to offer less likelihood towards VTE. A prospective cohort study by Eischer and colleagues showed a correlation between high hematocrit (RBC count) and recurrence of VTE in women [52]. A study by Harringa and associates demonstrated that there was no significant difference in the mean hemoglobin levels in PE vs. non-PE groups [53]. This finding does not appear to be documented in other literature. The data on the relationship between certain hematologic lab values and VTE are sparse. There have been some previous studies demonstrating that increased red cell distribution width (RDW) correlates to increased likelihood of VTE [54]. Byrnes and associates suggested that an increase in the RBC count may be associated with both arterial and venous thrombus formation through separate mechanisms [55]. In the setting of a known PFO, both of these scenarios could lead to increased risk of stroke. The increase in RBC count increases viscosity, which in turn, increases thrombosis. However, the same study highlighted a U-shaped relationship between the RBC count or hematocrit and odds of thrombosis. Therefore, the exact relationship between the RBC count and likelihood of thrombosis is not a simple one, and likely involves an unknown regulatory change in the balance of adenosine diphosphate (ADP, prothrombotic factor) or nitrous oxide (antithrombotic factor) secreted by the RBCs. Our finding tends to offer newer insight in this specific patient population.

Lastly, in our group of patients with PFOwoPE, we found a statistically significant increase in the association of alcohol use. While the relationship between alcohol use and likelihood of ischemic stroke is complex, a meta-analysis of the existing literature conducted by Larsson and colleagues suggested that high alcohol intake (i.e., more than two drinks per day) is associated with an increased risk of ischemic stroke [56]. Given that we could not obtain the quantification data of alcohol use in our study, we cannot determine the impact that alcohol consumption may have had in our results.

The major strength of our study was a fairly large sample size of patients with PFO with or without PE from one office location in our healthcare system. Subsequent follow-up of patient visits with the same group of healthcare providers allowed proper documentation of comorbid conditions in the electronic medical record. Nevertheless, our study had a few limitations, such as retrospective analysis allowed collection 
of only documented variables, which limited the correlation of precise timing of the key events, such as PE and stroke. The use of oral anticoagulant was significantly higher in the PFOwiPE group, which might have played a role in preventing stroke in this group, but the temporal relationship of stroke and PE was not clearly documented in many of the patient records.

We conclude that the association of ischemic stroke was similar in the patients who had PFO with or without PE. A higher BMI, lower LVEF and lower RBC count were associated with patients who had PFOwiPE compared to those without PE. Patients with PFO and PE had a higher association of CHF compared to the patients who had PFOwoPE.

\section{Acknowledgments}

The authors thank Christine Rickette RN (study coordinator) for her contribution to this study.

\section{Financial Disclosure}

None to declare.

\section{Conflict of Interest}

None to declare.

\section{Informed Consent}

Not applicable. This is a retrospective chart review study; hence the CUHC Institutional Review Board waived the need for informed consent.

\section{Author Contributions}

SR contributed to study design, data collection and manuscript writing. All authors contributed to data collection, and reviewed and edited the manuscript for the intellectual content. $\mathrm{KH}$ contributed to data analysis.

\section{Data Availability}

The authors declare that data supporting the findings of this study are available within the article.

\section{References}

1. Hara H, Virmani R, Ladich E, Mackey-Bojack S, Titus J, Reisman M, Gray W, et al. Patent foramen ovale: current pathology, pathophysiology, and clinical status. J Am Coll Cardiol. 2005;46(9):1768-1776.

2. Wu LA, Malouf JF, Dearani JA, Hagler DJ, Reeder GS,
Petty GW, Khandheria BK. Patent foramen ovale in cryptogenic stroke: current understanding and management options. Arch Intern Med. 2004;164(9):950-956.

3. Kerut EK, Norfleet WT, Plotnick GD, Giles TD. Patent foramen ovale: a review of associated conditions and the impact of physiological size. J Am Coll Cardiol. 2001;38(3):613-623.

4. American Heart Association. Patent Foramen Ovale (PFO). Available at: https://www.heart.org/en/healthtopics/congenital-heart-defects/about-congenital-heartdefects/patent-foramen-ovale-pfo. Accessed January 12, 2020.

5. Doyen D, Castellani M, Moceri P, Chiche O, Lazdunski R, Bertora D, Cerboni P, et al. Patent foramen ovale and stroke in intermediate-risk pulmonary embolism. Chest. 2014;146(4):967-973.

6. Goliszek S, Wisniewska M, Kurnicka K, Lichodziejewska B, Ciurzynski M, Kostrubiec M, Golebiowski M, et al. Patent foramen ovale increases the risk of acute ischemic stroke in patients with acute pulmonary embolism leading to right ventricular dysfunction. Thromb Res. 2014;134(5):1052-1056.

7. Clergeau MR, Hamon M, Morello R, Saloux E, Viader F, Hamon M. Silent cerebral infarcts in patients with pulmonary embolism and a patent foramen ovale: a prospective diffusion-weighted MRI study. Stroke. 2009;40(12):37583762.

8. Vindis D, Hutyra M, Sanak D, Kral M, Cechakova E, Littnerova S, Adam T, et al. Patent Foramen Ovale and the Risk of Cerebral Infarcts in Acute Pulmonary Embolism-A Prospective Observational Study. J Stroke Cerebrovasc Dis. 2018;27(2):357-364.

9. Konstantinides S, Geibel A, Kasper W, Olschewski M, Blumel L, Just H. Patent foramen ovale is an important predictor of adverse outcome in patients with major pulmonary embolism. Circulation. 1998;97(19):1946-1951.

10. Di Tullio MR, Sacco RL, Sciacca RR, Jin Z, Homma S. Patent foramen ovale and the risk of ischemic stroke in a multiethnic population. J Am Coll Cardiol. 2007;49(7):797-802.

11. Schmidt MR, Sondergaard L. Patent Foramen Ovale: A Villain in Pulmonary Embolism? Ann Intern Med. 2019;170(11):805-806.

12. Le Moigne E, Timsit S, Ben Salem D, Didier R, Jobic Y, Paleiron N, Le Mao R, et al. Patent Foramen Ovale and Ischemic Stroke in Patients With Pulmonary Embolism: A Prospective Cohort Study. Ann Intern Med. 2019; 170(11):756-763.

13. Tanislav C, Puille M, Pabst W, Reichenberger F, Grebe M, Nedelmann M, Kaps M, et al. High frequency of silent pulmonary embolism in patients with cryptogenic stroke and patent foramen ovale. Stroke. 2011;42(3):822-824.

14. Lapergue B, Decroix JP, Evrard S, Wang A, Bendetowicz D, Offroy MA, et al. Diagnostic yield of venous thrombosis and pulmonary embolism by combined CT venography and pulmonary angiography in patients with cryptogenic stroke and patent foramen ovale. Eur Neurol. 2015;74:69-72.

15. Bang OY, Lee MJ, Ryoo S, Kim SJ, Kim JW. Pat- 
ent foramen ovale and stroke-current status. J Stroke. 2015;17(3):229-237.

16. Zhang M, Tan S, Patel V, Zalta BA, Shmukler A, Levsky JM, Jain VR, et al. Patent foramen ovale in patients with pulmonary embolism: A prognostic factor on CT pulmonary angiography? J Cardiovasc Comput Tomogr. 2018;12(4):271-274.

17. Lamy C, Giannesini C, Zuber M, Arquizan C, Meder JF, Trystram D, Coste J, et al. Clinical and imaging findings in cryptogenic stroke patients with and without patent foramen ovale: the PFO-ASA Study. Atrial Septal Aneurysm. Stroke. 2002;33(3):706-711.

18. Foster PP, Boriek AM, Butler BD, Gernhardt ML, Bove AA. Patent foramen ovale and paradoxical systemic embolism: a bibliographic review. Aviat Space Environ Med. 2003;74(6 Pt 2):B1-64.

19. Kent DM, Thaler DE, Ro PESI. The Risk of Paradoxical Embolism (RoPE) Study: developing risk models for application to ongoing randomized trials of percutaneous patent foramen ovale closure for cryptogenic stroke. Trials. 2011;12:185.

20. De Castro S, Cartoni D, Fiorelli M, Rasura M, Anzini A, Zanette EM, Beccia M, et al. Morphological and functional characteristics of patent foramen ovale and their embolic implications. Stroke. 2000;31(10):2407-2413.

21. Anzola GP, Zavarize P, Morandi E, Rozzini L, Parrinello $\mathrm{G}$. Transcranial Doppler and risk of recurrence in patients with stroke and patent foramen ovale. Eur J Neurol. 2003;10(2):129-135.

22. Mas JL, Arquizan C, Lamy C, Zuber M, Cabanes L, Derumeaux G, Coste J, et al. Recurrent cerebrovascular events associated with patent foramen ovale, atrial septal aneurysm, or both. N Engl J Med. 2001;345(24):17401746.

23. Serena J, Marti-Fabregas J, Santamarina E, Rodriguez JJ, Perez-Ayuso MJ, Masjuan J, Segura T, et al. Recurrent stroke and massive right-to-left shunt: results from the prospective Spanish multicenter (CODICIA) study. Stroke. 2008;39(12):3131-3136.

24. Homma S, Sacco RL, Di Tullio MR, Sciacca RR, Mohr JP, Investigators PFOiCSS. Effect of medical treatment in stroke patients with patent foramen ovale: patent foramen ovale in Cryptogenic Stroke Study. Circulation. 2002;105(22):2625-2631.

25. Poulin MF, Kavinsky CJ. The approval of PFO closure in the United States. Pract Neurol. 2018;1:13-38.

26. Najib K, Heckle M, Goubran S, Mehta R, Goswami R, Khouzam RN. Paradoxical emboli following a pulmonary embolus in the presence of a patent foramen ovale. Ann Transl Med. 2018;6(1):21.

27. He D, Li Q, Xu G, Hu Z, Li X, Guo Y, Xu S, et al. Clinical and imaging characteristics of PFO-related stroke with different amounts of right-to-left shunt. Brain Behav. 2018;8(11):e01122.

28. Overell JR, Bone I, Lees KR. Interatrial septal abnormalities and stroke: a meta-analysis of case-control studies. Neurology. 2000;55(8):1172-1179.

29. Kraywinkel K, Jauss M, Diener HC, Weimar C. [Patent foramen ovale, atrial septum aneurysm, and stroke. An examination of the status of recent evidence]. Nervenarzt. 2005;76(8):935-942.

30. Sipola P, Hedman M, Jakala P, Karjalainen AM, Peuhkurinen K, Manninen H, Vanninen R. Prevalence of pulmonary embolism in patients with suspected cardioembolic ischemic stroke. J Thorac Imaging. 2011;26(1):32-35.

31. Tanislav C, Puille M, Grebe M, Sieweke N, Allendorfer J, Pabst W, Kaps M, et al. Factors associated with shunt dynamic in patients with cryptogenic stroke and patent foramen ovale: an observational cohort study. BMC Cardiovasc Disord. 2011;11:54.

32. Sun YP, Homma S. Patent Foramen Ovale and Stroke. Circ J. 2016;80(8):1665-1673

33. Yasaka M, Otsubo R, Oe H, Minematsu K. Is stroke a paradoxical embolism in patients with patent foramen ovale? Intern Med. 2005;44(5):434-438.

34. Stein PD, Beemath A, Olson RE. Obesity as a risk factor in venous thromboembolism. Am J Med. 2005;118(9):978980 .

35. Ageno W, Becattini C, Brighton T, Selby R, Kamphuisen PW. Cardiovascular risk factors and venous thromboembolism: a meta-analysis. Circulation. 2008;117(1):93102.

36. Kabrhel C, Varraso R, Goldhaber SZ, Rimm EB, Camargo CA. Prospective study of BMI and the risk of pulmonary embolism in women. Obesity (Silver Spring). 2009;17(11):2040-2046.

37. Linnemann B, Zgouras D, Schindewolf M, Schwonberg $\mathrm{J}$, Jarosch-Preusche M, Lindhoff-Last E. Impact of sex and traditional cardiovascular risk factors on the risk of recurrent venous thromboembolism: results from the German MAISTHRO Registry. Blood Coagul Fibrinolysis. 2008;19(2):159-165.

38. Borch KH, Braekkan SK, Mathiesen EB, Njolstad I, Wilsgaard T, Stormer J, Hansen JB. Abdominal obesity is essential for the risk of venous thromboembolism in the metabolic syndrome: the Tromso study. J Thromb Haemost. 2009;7(5):739-745

39. Rosenfeld HE, Tsokos M, Byard RW. The association between body mass index and pulmonary thromboembolism in an autopsy population. J Forensic Sci. 2012;57(5):1336-1338.

40. Darvall KA, Sam RC, Silverman SH, Bradbury AW, Adam DJ. Obesity and thrombosis. Eur J Vasc Endovasc Surg. 2007;33(2):223-233.

41. Song B, Pei L, Deng W, Fischer L, Chou I, CamargoFaye E, McMullin D, et al. Increased body mass index is associated with hereditary hypercoagulable state of PFOrelated cryptogenic stroke. Stroke. 2018;49:S1.

42. Howell MD, Geraci JM, Knowlton AA. Congestive heart failure and outpatient risk of venous thromboembolism: a retrospective, case-control study. J Clin Epidemiol. 2001;54(8):810-816.

43. Jug B, Vene N, Salobir BG, Sebestjen M, Sabovic M, Keber I. Procoagulant state in heart failure with preserved left ventricular ejection fraction. Int Heart J. 2009;50(5):591600.

44. Dries DL, Rosenberg YD, Waclawiw MA, Domanski MJ. Ejection fraction and risk of thromboembolic 
events in patients with systolic dysfunction and sinus rhythm: evidence for gender differences in the studies of left ventricular dysfunction trials. J Am Coll Cardiol. 1997;29(5):1074-1080.

45. Zhu R, Hu Y, Tang L. Reduced cardiac function and risk of venous thromboembolism in Asian countries. Thromb J. 2017;15:12.

46. Samama MM. An epidemiologic study of risk factors for deep vein thrombosis in medical outpatients: the Sirius study. Arch Intern Med. 2000;160(22):3415-3420.

47. Beemath A, Stein PD, Skaf E, Al Sibae MR, Alesh I. Risk of venous thromboembolism in patients hospitalized with heart failure. Am J Cardiol. 2006;98(6):793-795.

48. Piazza G, Goldhaber SZ. Pulmonary embolism in heart failure. Circulation. 2008;118(15):1598-1601.

49. Ray JG, Rosendaal FR. The role of dyslipidemia and statins in venous thromboembolism. Curr Control Trials Cardiovasc Med. 2001;2(4):165-170.

50. McColl MD, Sattar N, Ellison J, Tait RC, Walker ID, Packard CJ, Greer IA. Lipoprotein (a), cholesterol and triglycerides in women with venous thromboembolism. Blood Coagul Fibrinolysis. 2000;11(3):225-229.
51. Morelli VM, Lijfering WM, Bos MHA, Rosendaal FR, Cannegieter SC. Lipid levels and risk of venous thrombosis: results from the MEGA-study. Eur J Epidemiol. 2017;32(8):669-681.

52. Eischer L, Eichinger S, Kyrle PA. The risk of recurrence in women with venous thromboembolism while using estrogens: a prospective cohort study. J Thromb Haemost. 2014;12(5):635-640.

53. Harringa JB, Bracken RL, Nagle SK, Schiebler ML, Patterson BW, Svenson JE, Repplinger MD. Anemia is not a risk factor for developing pulmonary embolism. Am J Emerg Med. 2017;35(1):146-149.

54. Hammons L, Filopei J, Steiger D, Bondarsky E. A narrative review of red blood cell distribution width as a marker for pulmonary embolism. J Thromb Thrombolysis. 2019;48(4):638-647.

55. Byrnes JR, Wolberg AS. Red blood cells in thrombosis. Blood. 2017;130(16):1795-1799.

56. Larsson SC, Wallin A, Wolk A, Markus HS. Differing association of alcohol consumption with different stroke types: a systematic review and meta-analysis. BMC Med. 2016;14(1):178. 